\title{
Effects of Different Hormonal Concentrations and Culture Medium on Multiplication and Rooting of Stage II Banana (Musa cavendishii)
}

\author{
Yapa Mudiyanselage A.M. WIJERATHNA ${ }^{1 *}$, Herath Mudiyanselage P.C. KUMARIHAMI ${ }^{2}$ \\ IThe Hebrew University of Jerusalem, Robert H. Smith Faculty of Agriculture, Food and Environment, Division for International Studies, Israel; \\ akila.mw.yapa@gmail.com ("correspondingauthor) \\ 2Jeju National University, Faculty of Bioscience \& Industry,Jeju690-756, Korea; p.chamidha@yahoo.com
}

\begin{abstract}
Banana (Musa spp.) breeding has been a slow developing process, due to the absence of seeds and low propagation rates. Selection of valuable cultivars and micro-propagation are promising techniques to accelerate the cultivation process. Therefore, callus culture was carried out, aiming the establishment of plant regeneration protocol that might be used in banana breeding programs. Sword suckers were used as explants, whereas vigorously grown apical shoots obtained from initial in vitro germinated seedlings were sub-cultured in six different MS media at pH 5.8, supplemented with MS Minerals + MS Vitamins + 36.7 FeNaEDTA + 3\% sucrose + 0.65\% agar (+) different hormonal conditions. Among the different concentrations tested within the experiment, MS Media (MS Minerals + MS Vitamins + $36.7^{*} \mathrm{FeNaEDTA}+3 \%$ sucrose $+0.65 \%$ agar) with hormones $1 \mathrm{TDZ}+10 \mathrm{IBA}$ showed the highest shoot proliferation and a high rate of multiplication. Even so, there were no significant differences observed for root initiation and plantlet establishment among the six tested medium.
\end{abstract}

Keywords: benzylaminopurine, thidiazuron, indole-3-butyric acid, kinetin, micro propagation, Musa cavendishii

\section{Introduction}

Edible bananas (Musa spp.) are the major staple food for rural and urban consumers in the tropical and subtropical countries and an important source of rural income (Rowe, 1981). The commercial cultivars propagated in Taiwan belong to the Cavendish subgroup (AAA). Banana has been conventionally propagated through suckers, which represent a time-consuming method, with lower rates of multiplication. To overcome these aspects, the standardized protocols for shoot-tip culture have been commercially exploited by various tissue culture protocols. Tissue culture has been proven as a potential technology to produce millions of identical plantlets, which are disease free and true to parental type. Through this technology, from a single shoot tip or an axillary bud (Musa sapientum cv. 'Sagar'), a large quantity of uniform and disease free plants, with good genetic potential, were produced within a short time (Vuylsteke and Langhe, 1985; Wong, 1986).

Banana is one of the most intensely micropropagated crop, with specific protocols achieved during the past two decades. However, a large number of banana genotypes need to be screened for commercial micropropagation and genetic improvement. Different explants have been used for banana and plantain propagations. In vitro propagation of banana using shoot tips has been reported for many commercial cultivars (Kulkarni et al., 2004;
2006). Banerjee and De Langhe (1985) used shoot tips, while other researchers choose floral apices for en masse multiplication (Cronauer and Krikorian, 1984; Cronauer and Krikorian, 1985; Azad and Amin, 2001).

An important factor affecting the efficiency of micropropagation system is the rate of multiplication. It has been observed that banana multiplication rate is genotypic dependent, whereas variable behavior has been observed among cultures initiated from the same banana genotypes cultured in vitro (Israeli et al., 1995; Mendes et al., 1996).

The multiplication rate of adventitious buds under the influence of Benzylaminopurine (BA) is one of the determining factors influencing the efficiency of the micropropagation system. The most commonly used cytokinin is Benzylaminopurine (BA), at a range of 2 to 5 $\mathrm{mg} / \mathrm{L}$, often in combination with an auxin, Indole-3butyric acid (IBA), at a concentration of 0.1 to $0.2 \mathrm{mg} / \mathrm{L}$ (Vuylsteke, 1998). Also Thidiazuron (TDZ) has been considered an active phenylureas, having cytokinin-like activities.

The present work was planned to study the multiplication rates of banana shoot tips under in vitro conditions during successive sub-cultures, using different medium conditions. Different concentrations of cytokinins viz. BA, kinetin, TDZ and auxin, IBA were used in MS medium to test the best multiple shoot proliferation from the explants and the formation of 
roots from shoots. The present study was undertaken with the following objectives: i) to study the effects of $\mathrm{BA}$, kinetin, TDZ and IBA growth regulators on in vitro meristem and shoot proliferation of banana cv. 'Cavendishii', ii) to determine the effect of BA, kinetin, TDZ and IBA growth regulators and their concentration required for in vitro root development of banana plantlets.

\section{Materials and Methods}

\section{Preparation of explants (Stage I multiplication)}

Sword suckers were used as explants for the hereby experiment. Leaf sheaths and bracts were removed to a size of $1 \times 1 \mathrm{~cm}$. The explants were sterilized two times in $10 \%$ Clorox $(\mathrm{NaOCl})$ for $15 \mathrm{~min}$ and then were rinsed in sterile distilled water for three times. They were longitudinally cut into four pieces, every piece with an apical meristem. Hereafter, the prepared explants were cultured in semi-solid Murasige and Skoog (MS) (1962) supplemented with $5 \mathrm{ppm} \mathrm{BA}$ and $\mathrm{pH}$ at 5.8. All cultures were maintained at $25-28{ }^{\circ} \mathrm{C}$ with $1,500-2,000$ Lux of light and $80-85 \%$ RH. Observations were made on plant proliferation and the number of shoots regenerated.

\section{Establishment of sub-cultures (stage II multiplication)}

After 21 days, vigorously grown apical shoots obtained from initial in vitro germinated seedlings were sub-cultured in six different MS media at $\mathrm{pH} 5.8$, supplemented with MS Minerals + MS Vitamins + $36.7^{*} \mathrm{FeNaEDTA}+3 \%$ sucrose $+0.65 \%$ agar $(+)$ different hormonal conditions, as follows:

- Medium 1 (M1): (+) 10 IBA + 10 Kinetin,

- Medium 2(M2): (+) $10 \mathrm{BA}$,

- Medium 3 (M3): (+) $10 \mathrm{BA}+1 \mathrm{TDZ}$,

- Medium 4 (M4): no hormones,

- Medium 5(M5): (+) 1 TDZ

- Medium 6 (M6): (+) 1 TDZ + 10 IBA.

All cultures were maintained at $25-28^{\circ} \mathrm{C}$ with 1,500 2,000 Lux of light and $80-85 \% \mathrm{RH}$, for four weeks. Observations were made on plant proliferation and the number of shoots and roots regenerated.

\section{Establishment of rooting (stage III)}

Plantlets were transferred to new medium consisting viz. MS Minerals + MS Vitamins + 36.7 ${ }^{*} \mathrm{FeNaEDTA}+$ 2 IBA $+3 \%$ sucrose $+0.65 \%$ agar $(\mathrm{pH} 5.8)$. After 14 days, observations and data on shoot initiation and number of shoots developed were recorded.

\section{Data analysis}

The data were systematized using analysis of variance (ANOVA). The significant effect of each factor on number of roots and shoots were separated by Tukey's pair wise comparison post hoc (Least Significance Difference) test at $5 \%$ level of significance by using JMP statistical software.

\section{Results}

The creamy white apical meristem slices turned green when placed in MS medium supplemented with $5 \mathrm{ppm}$

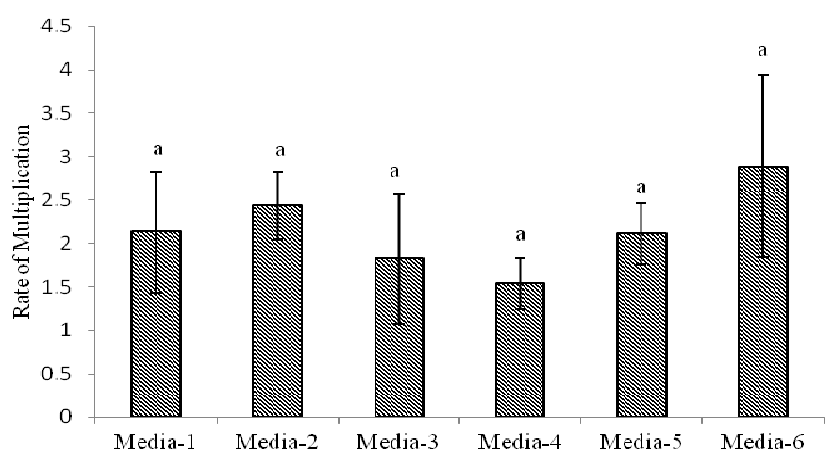

Fig. 1. Rate of shoots multiplication at $4^{\text {th }}$ week after sub-culturing Means with different letters in each column are significantly different at $\mathrm{P}<0.05$ at 95\% confidence level.

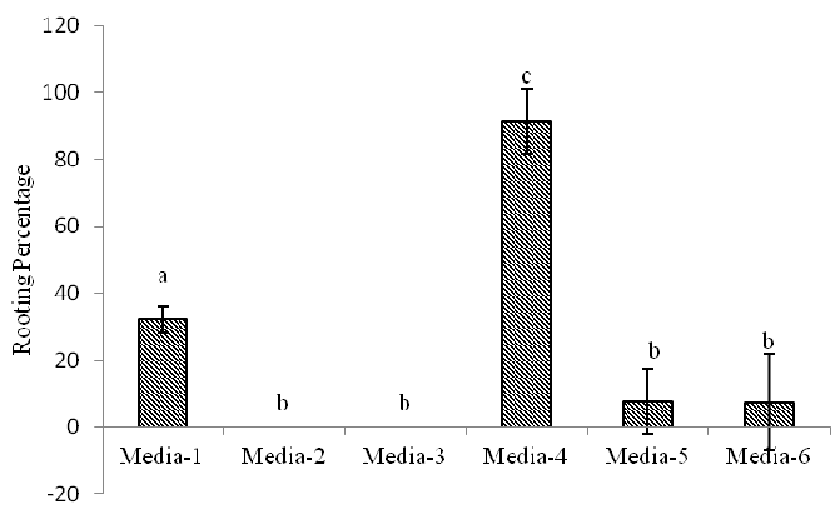

Fig. 2. Root elongation in harvested cuttings at $4^{\text {th }}$ week after subculturing

Means with different letters in each column are significantly different at $\mathrm{P}<0.05$ at $95 \%$ confidence level.

BA and small shoot buds were visible within 21 days of the experiment. The shoots obtained within stage-I multiplication were transferred on six different media and cultivated for 4 weeks for experimental data observations.

\section{Stage II multiplication}

After the first four weeks of culturing, the external leaf primordia of explants, which were initially creamy white, turned green. The size of the explants also increased, while blackening was observed at the base of the explants. No statistical significant differences were observed among the six different media (Fig. 1), but there was an evident higher rate of multiplication in Medium 6, which was supplemented with 1 TDZ + 10 IBA.

According to the statistical analysis, root elongation response was significantly different among the hormonal treatments. Medium 4, which did not contained hormones, performed well with regards to the rooting (Fig. 2).

\section{Stage III root initiation and plantlet establishment}

The elongated shoots were transferred to rooting medium consisting viz. MS Minerals + MS Vitamins + $36.7^{*} \mathrm{FeNaEDTA}+2 \mathrm{IBA}+3 \%$ sucrose $+0.65 \%$ agar (pH 5.8); after 14 days in this phase of the experiment, all explants had similar shooting and rooting and there were no significant differences among the shoots that originated from the six different media tested (Figs. 3 and $4)$. 


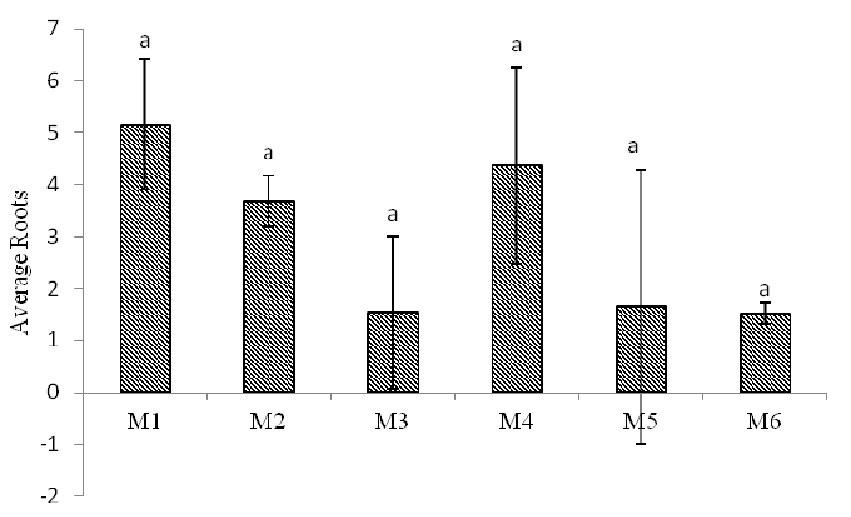

Fig. 3. Average roots per plantlet obtained after two weeks of subculturing

\section{Discussion}

After the first 21 days, the explants (suckers) become greenish in color and the multiple shoots arised from the base of the suckers. These shoots were cut at the base, separated and placed in the six different media within the experiment. After 4 weeks of sub-culture, multiple shoots were formed from the inoculated shoots (Fig. 5). Then these shoots were separated and placed in a fresh medium for rooting.

The blackening of the explants may be due to secretion of phenolic compounds (Abdelwahd et al., 2008). Sub-cultured, some meristems suffered damages and this may be a reason for obtaining statistically nonsignificant results.

Multi cultures were further divided and transferred to shooting media which were composed with auxins (IBA) to obtain the elongation of plantlets. In this stage, leaves developed and the whole plants grow up to 4 to $5 \mathrm{~cm}$.

By comparison, Medium 6 (1 TDZ + 10 IBA) gave the highest axillary shoots proliferation from the callus. The result showed that TDZ was stable and more active at lower concentrations than the adenine-type cytokinins kinetin. Herman (1997) described that TDZ induced greater proliferation of axillary shoots compared with other cytokinins at low concentrations (less than $1 \mu \mathrm{M}$ ). At higher concentrations, TDZ stimulated the formation of callus, shoots or somatic embryos.

This intense activity of TDZ may affect the explants' development during the rooting, since it was observed in the current experiment that some clusters of shoots developed from shoots obtained on Medium 5, during rooting stage. According to Huettman and Preece (1993), shoots became stunted and clumps of globular buds occurred with TDZ supplemented medium and suppressed the elongation of shoots; these could however return to normal sizes after being transferred to an elongation medium containing a lower TDZ level. This may be the reason for the similar situation occurred in propagules obtained from Medium 5.

Medium supplemented MS Minerals + MS Vitamins $+36.7^{*} \mathrm{FeNaEDTA}+3 \%$ sucrose $+0.65 \%$ agar rendered the highest root elongation. Since there were no significant differences observed in shoot proliferation among the tested mediums within the current

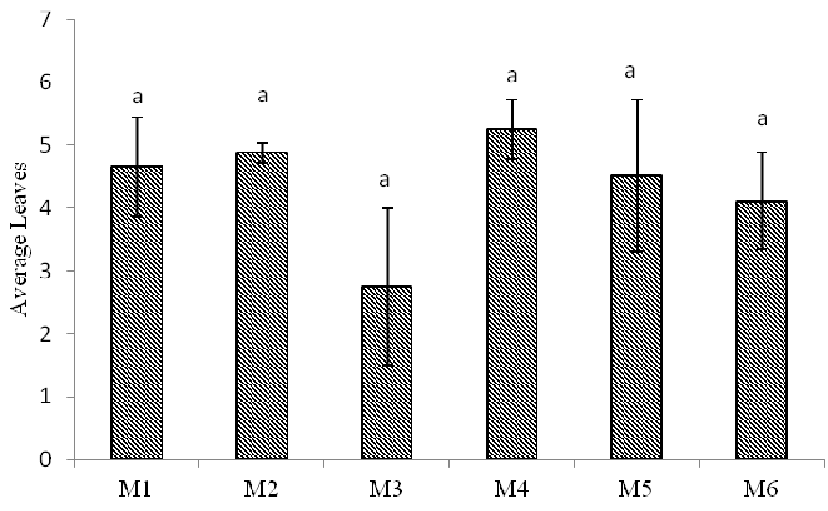

Fig. 4. Average leaves per plantlet obtained after two weeks of subculturing

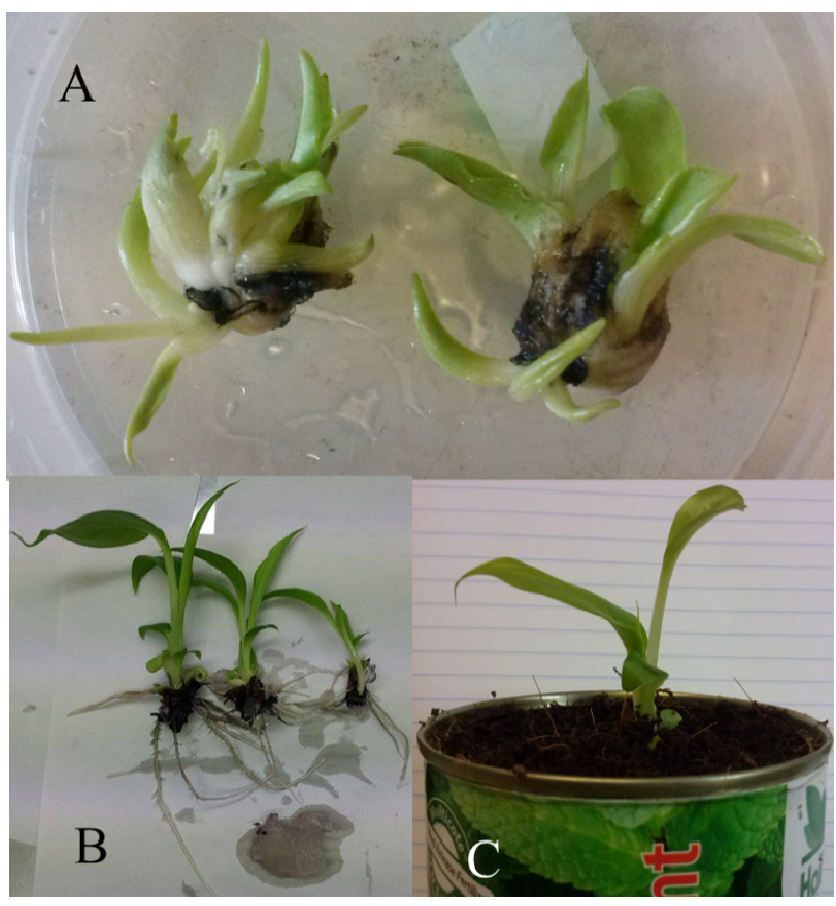

Fig. 5. Stages of banana plantlets micropropagation from shoot-tip meristems explants:

A. Clusters of shoots developed from shoots, during rooting stage; $\mathbf{B}$. Elongated multiple shoots with profuse leaves and roots, on rooting medium; C. Regenerated plant in pot containing coir dust

experiment, further experiments are needed for the establishment of proper shoot multiplication protocols, starting from the plant house grown callus cuttings.

\section{Conclusions}

The proposed strategy for Cavendish banana micropropagation consists of using 1 TDZ +10 IBA supplemented medium to induce adventitious shoots from the explants (meristematic region of the sucker). Finally, bud clusters should be sub-cultured in medium consisting viz. MS Minerals + MS Vitamins + $36.7^{*}$ FeNaEDTA +2 IBA $+3 \%$ sucrose $+0.65 \%$ agar ( $\mathrm{pH}$ 5.8) for root elongation of the new formed shoots. 
72

\section{Acknowledgement}

The authors would like to acknowledge Dr. E. Khayat for giving valuable suggestions for the experiment.

\section{References}

Abdelwahd R, Hakam N, Labhilili M, Udupa SM (2008). Use of an adsorbent and antioxidants to reduce the effects of leached phenolics in in vitro plantlet regeneration of faba bean. African Journal of Biotechnology 7(8):997-1002.

Azad MAK, Amin MN (2001). Rapid clonal propagation of banana (Musa sp.) using in vitro culture of floral bud apex. Plant Tissue Culture 11(1):1-9.

Banerjee N, De Langhe E (1985). A tissue culture technique for rapid clonal propagation and storage under minimal growth conditions of Musa (Banana and Plantain). Plant Cell Reports 4:351-354.

Cronauer SS, Krikorian AD (1984a). Rapid multiplication of banana and plantains by in vitro shoot tip culture. HortScience Journal 19(2):234-235.

Cronauer SS, Krikorian AD (1985a). Aseptic multiplication of banana from excised floral apices. HortScience 20:770-771.

Herman EB (1995). Recent advances in plant tissue culture. Agritech Consultants.

Huetteman CA, Preece JE (1993). Thidiazuron: a potent cytokinin for woody plant tissue culture. Plant Cell, Tissue and Organ Culture 33:105-119.

Israeli Y, Lahav E, Reuveni O (1995). In vitro culture of bananas. In: Bananas and plantains Springer Netherlands pp 147-178.
Kulkarni VM, Suprasanna P, Ganapathi TR, Bapat VA, Rao PS (2004). Differential effect of genome and cytokinins on shoottip cultures of Indian banana cultivars. Physiology and Molecular Biology of Plants 10:75-81.

Kulkarni VM, Suprasanna P, Bapat VA (2006). Plant regeneration through multiple shoot formation and somatic embryogenesis in a commercially important and endangered Indian banana cv. Rajeli. Current Science 90:842-846.

Mendes BMJ, Mendes FJ, Neto AT, Demetrio CGB, Puske OR (1996). Efficacy of banana plantlet production by micropropagation. Pesquisa Agropecuária Brasileira 31:863867.

Murashige T, Skoog F (1962). A revised medium for rapid growth and bioassays with tobacco tissue cultures. Physiologia Plantarum 15:473-479.

Rowe PR (1981). Breeding an intractable crop. In: Rachie KO, Lyman JM (Eds). Banana: genetic engineering for crop improvement. Working Papers. The Rockefeller Foundation pp 66-84.

Vuylsteke DR (1998). Shoot-tip culture for the propagation, conservation and exchange of Musa germplasm. International Institute of Tropical Agriculture, Ibadan, Nigeria.

Vuylsteke D, De Langhe EAL (1985). Feasibility of in vitro propagation of bananas and plantains. Tropical Agriculture (Trinidad) 62:323-328.

Wong WC (1986). In vitro propagation of banana (Musa spp.): Initiation, proliferation and development of shoot tip cultures on defined media. Plant Cell, Tissue and Organ Culture 6:159-166. 